\title{
Evaluation des facteurs de risque susceptibles de produire des infections nosocomiales chez le sujet âgé hospitalisé dans les services de médecine interne et maladie infectieuse du centre hospitalier de Kiffa Mauritanie.
}

\section{Evaluation of risk factors linked to nosocomial infections in elderly hospitalized patients in the internal medicine and infectious diseases units in Kiffa hospital, Mauritania.}

Boushab MB ${ }^{1}$, Savadogo $\mathrm{M}^{2}$, Ould Cheikh Melaïnine $\mathrm{ML}^{3}$, Darnycka BM${ }^{4}$, Traore $\mathrm{AM}^{5}$, Fall-Malick FZ .

1. Médecine interne et Maladies Infectieuses, Centre Hospitalier de Kiffa, Assabai, Mauritanie

2. Service des Maladies Infectieuses, CHU Yalgado Ouédraogo, Ouagadougou, Burkina Faso ;

3. Ambulatory Treatment Center, National Hospital Center of Nouakchott, Mauritanie

4. Health Security and Emergency Officer, WHO World Health Emergency Programme, Mauritania

5. Service des Maladies Infectieuses, Hopital du Point G, Bamako, Mali.

6. Institut National d'Hépato-Virologie, Faculté de Médecine de Nouakchott, Mauritanie

Auteur correspondant: Boushab Mohamed Boushab, Médecine Interne et Maladies Infectieuses, Centre Hospitalier de Kiffa, Assaba, Mauritanie Email : bboushab@gmail.com,

\section{Résumé}

Objectif : Déterminer les facteurs de risque des infections nosocomiales (IN) chez les patients âgés hospitalisés dans le Service de Médecine Interne et Maladies Infectieuses du Centre Hospitalier de Kiffa. Patients et Méthodes : Il s'agissait d'une étude transversale et descriptive, menée du $1^{\mathrm{er}}$ janvier au 31 mai 2017. Tous les patients hospitalisés depuis au moins $48 \mathrm{~h}$ étaient inclus dans l'étude. Résultat : Au total 73 sujets âgés ont été hospitalisés soit une proportion de $24,17 \%$. Le sex-ratio M/F était de 2,31 et l'âge moyen était de $75 \pm 10$ ans avec des extrêmes de (60-90 ans). La majorité de nos patients (58,9\%) avait une hospitalisation supérieure à sept jours. La fièvre associée à l'altération de l'état général étaient le principal motif de consultation. Les infections de l'appareil respiratoire représentaient $49,7 \%$ suivi des appareils urogénital $(28,8 \%)$, digestif $(16,4 \%)$ et cutanéomuqueux $(6,8 \%)$. Les facteurs de susceptibilité aux infections IN étaient liées à l'âge $(p=0,00062)$, à la perte d'autonomie $(p=0,0004)$, altération de l'état général ( $p=0,0004)$, aux portes d'entrées $(p=0,0001)$, aux poly pathologies $(p=0,02)$ et à la poly médication $(p=0,04)$. La létalité globale était $15,1 \%$ malgré a la prise en charge. Conclusion : Ce travail permet de penser que les sujets âgés dans un tel contexte sont largement exposés au risque élevé d'IN, même si, pour des raisons techniques, les examens microbiologiques n'ont pas été effectués dans notre population d'étude.

Mots clés : Facteurs de risque, Pathologies gériatriques, Personnes âgées, Mauritanie.

\begin{abstract}
:
Objective: To determine the risk factors for nosocomial infections (NSIs) in elderly patients hospitalized in the Department of Internal Medicine and Infectious Diseases at the Kiffa Hospital Center. Patients and Methods: This was a cross-sectional and descriptive study conducted from January 1 to May 31, 2017. All patients hospitalized for at least 48 hours were included in the study. Result: A total of 73 elderly subjects were hospitalized for a prevalence of $24.17 \%$. The M / F sex ratio was 2.31 and the average age was $75 \pm 10$ years with a range of (60-90 years). The majority of our patients $(58.9 \%)$ had higher hospitalization was seven days. Fever associated with poor general condition were the main reason for consultation. Site respiratory infections accounted for $49.7 \%$ site monitoring urogenital $(28.8 \%)$, gastrointestinal (16.4\%) and skin (6.8\%). Susceptibility factors were related to infections IN age $(p=0.00062)$, loss of autonomy $(p=0.0004)$, impaired general condition $(p=0.0004)$, the gates of ' entries $(p=0.0001)$, the pathologies poly $(p=0.02)$ and poly medication $(p$ $=0.04)$. Overall mortality was $15.1 \%$ despite a support. Conclusion: This work suggests that the elderly subjects in this context are widely exposed to high IN risk, even if, for technical reasons, microbiological examinations were not performed in our study population.
\end{abstract}

Keywords: Risk factors, geriatric pathologies, old people, Mauritania 


\section{Introduction}

Les personnes âgées, dont le nombre croit régulièrement partout dans le monde, sont particulièrement exposées aux infections communautaires et surtout nosocomiales [1]. L'altération des mécanismes de défenses liées au processus de vieillissement et la perte d'autonomie ou l'immobilisation relative des patients induisent des modifications physiologiques qui prédisposent l'individu âgé à l'infection [1, 2,3]. Au-delà du terrain et de la pathologie, les soins au patient dépendant constituent un risque nosocomial supplémentaire [3]. De façon conventionnelle, une infection est dite nosocomiale lorsqu'elle survient 48 heures, au moins, après l'admission dans un service hospitalier. Elle s'oppose aux infections communautaires qui surviennent chez des personnes non hospitalisées et qui vivent à leur domicile [4].Cependant, les facteurs de cette susceptibilité sont multiples et leur responsabilité respective est difficile à établir [1] .Ce travail avait pour objectif principal de déterminer la proportion des personnes âgées hospitalisées dans le service de Médecine Interne et Maladies Infectieuses du centre hospitalier de Kiffa, d'évaluer les facteurs de risque des IN dans le but de sensibiliser l'ensemble du personnel hospitalier sur la réalité de ces infections.

\section{Patients et méthodes :}

Notre étude est épidémiologique, transversale avec collecte prospective portant sur les données des patients âgés hospitalisés pendant au moins $48 \mathrm{~h}$ entre le $1^{\text {er }}$ janvier et le 31 mai 2017. Ont été prises en compte les infections nosocomiales actives, c'est-à-dire les infections nosocomiales en cours de traitement antiinfectieux par voie générale. Les définitions des infections nosocomiales retenues ont été celles des «100 recommandations »_[4]. La définition du sujet âgé utilisée pour cette étude est celle de 1'Organisation Mondiale de la Santé (OMS): qui stipule une population hétérogène âgée de 60ans et plus [5]. Un questionnaire préétabli à l'occasion de cette étude a été complété pour chaque patient inclus. Nous avons procédé à un échantillonnage exhaustif de tous les cas répondant à la définition de personne âgée et ayant accepté de participer librement à notre étude. Les informations, recueillies sur les patients âgés comprennent les données sociodémographiques (âge, sexe, provenance), les données générales et cliniques. Le site de l'infection, la durée de séjour, le décès étaient aussi collectés. Les données microbiologiques n'étaient pas disponibles car aucun examen biologique à la recherche des microorganismes n'a été effectué ceci était dû à l'insuffisance du plateau technique. L'hôpital ne dispose pas d'un laboratoire de bactériologie. En cas de doute par rapport à la classification de l'infection nosocomiale, et plus particulièrement lorsque des doutes existaient sur la présence de facteurs de risque tels qu'une sonde urinaire ou un cathéter vasculaire, le malade était réexaminé et son état discuté entre le médecin traitant et les confrères de l'hôpital afin de clarifier et de classer correctement l'infection. La saisie et l'analyse des données ont été effectuées avec le logiciel Epi info version 6.04. La comparaison des variables qualitatives a été effectuée en utilisant le test du Khi 2, considéré significative pour une valeur de $p$ inférieur à 0,05. Par ailleurs, le même seuil est retenu pour $\mathrm{p}$ dans l'étude des facteurs de risque d'IN par rapport à la durée de l'hospitalisation et pour laquelle nous avons utilisé le test exact de Fischer lorsque l'effectif théorique était inférieur à 5.

\section{Résultats :}

Durant la période de l'étude, 73 patients âgés ont été hospitalisés dans le service de Médecine Interne et Maladies Infectieuses du centre hospitalier régional d'Kiffa, soit une proportion de 24,17\% (73/302 des admissions). L'âge moyen des patients était de $75 \pm 10$ ans avec des extrêmes de 60 et 90 ans. Les tranches d'âge les plus représentatives dans notre groupe d'étude étaient les suivantes : 6070 ans (60,3\% des cas), 71-80 ans (31,5\% des cas) et $81-90$ ans $(8,2 \%$ des cas $)$. Le sex-ratio homme/femme était de 2,31 cependant l'IN avaient concerné en majorité le sexe masculin (51/73 soit $69,9 \%)$. Plus de la moitié des enquêtés $(68,4 \%)$ provenait du milieu rural c'est-à-dire hors de la ville de Kiffa (Tableau I). Parmi nos patients, 15 avaient des antécédents de tuberculose, 10 hypertendus, sept hémiplégiques, six prostatectomisés, cinq porteurs chroniques d'AgHbs (Hépatite B chronique), quatre asthmatiques, deux diabétiques, deux insuffisants rénaux et un cas de fibrose pulmonaire. Les facteurs de susceptibilité aux infections étaient dominés par perte d'autonomie $(67,1 \%)$, suivie de l'AEG $(63 \%)$, des portes d'entrées $(53,4 \%)$, de la poly médication $(46,6 \%)$ et les poly pathologies $(23,3 \%)$. Globalement, les signes cliniques étaient dominés par la fièvre associée, l'altération de l'état général (AEG). Les principaux sites de l'infection étaient: respiratoire $(49,7 \%)$, urogénital $(28,8 \%)$, digestif $(16,4 \%)$ et enfin cutanéomuqueux $(6,8 \%)$ (Tableau I). La majorité de nos patients $(58,9 \%)$ avaient une durée $\mathrm{d}$ 'hospitalisation supérieure à sept jours tandis que $41,1 \%$ avaient une hospitalisation inférieure à sept jours. Les facteurs de risque d'IN étaient l'âge $(p=0,0062)$, la perte d'autonomie $(p=0,0004)$, l'AEG $(p=0,0004)$, les portes d'entrées $(p=0,0001)$, les poly pathologies $(p=0,02)$ et la poly médication $(p=0,04)$ ont été notés dans le tableau II. 


\section{Discussion :}

Le défi représenté par les IN n'épargne actuellement aucun pays mais la lutte quotidienne pour maintenir une exigence de propreté ne dépend plus simplement des moyens engagés. Elle nécessite surtout un réel changement de mentalité vis-à-vis de l'hospitalisme infectieux et souligne que la connaissance seule du risque infectieux n'est pas suffisante pour modifier les pratiques de soins [6]. Les patients âgés posent des problèmes d'ordre diagnostique, mais également accumulent les facteurs prédisposant à l'infection [1-3,7]. En effet, 73 sujets âgés ont été hospitalisés dans le service de médecine interne du centre hospitalier d'Kiffa, soit un pourcentage de 24,17\% (73/302 admissions). L'effectif réduit de cette population est liée d'une part a la faible espérance de vie en Mauritanie et d'autre part au refus habituel de celle-ci à consulter voire à se faire hospitaliser à l'hôpital. Aussi, la part importante des états morbides mal définis où la multi morbidité observées expliqueraient 1'orientation des patients âgés vers à un service d'urgence plutôt qu'un service spécialisé [8]. Parmi nos patients, la tranche d'âge la plus touchée était celle comprise entre $60-70$ ans (60,3\% des cas) avec un âge moyen de 75 ans \pm 10 . Dans la littérature actuelle, peu d'enquêtes ont étudié cette population. Les chiffres habituellement rapportés étant souvent supérieurs à $70 \%$ [9,10]. En effet, les enquêtes réalisées dans les autres pays étudient des populations de 65 ans et plus (ce qui correspond à la définition de la personne âgée par l'OMS). La plupart ont une moyenne d'âge d'environ 80 ans par rapport aux populations totales recueillies [912]. Notre étude a limité l'âge de la population gériatrique à 60 ans et plus. Cette limite d'âge reste en partie arbitraire, d'autant plus que c'est l'âge physiologique qui est surtout pris en compte pour les stratégies thérapeutiques. Son début est fixé à 60ans par 1'OMS [5], ce qui serait raisonnable pour l'Afrique du fait de la durée de vie restreinte [8]. Le sex-ratio homme/femme était de 2,31 mais l'infection avaient concerné en majorité le sexe masculin (51/73 soit $69,9 \%)$. En milieu hospitalier africain d'une façon générale, comme dans notre série d'étude, le sexe masculin est prédominant. Cette prédominance masculine s'explique, que l'homme chef de famille décide de son hospitalisation et de celle des autres membres de la famille [5]. Globalement les infections du site respiratoire était fortement représenté soit 49,7\% suivi du site urogénital $(28,8 \%)$, digestif $(16,4 \%)$ et cutanée $(6,8 \%)$. Ces résultats corroborent les données de la littérature [2, 7,11]. Plusieurs symptômes tels que l'anorexie, la perte de poids, l'apparition d'un état confusionnel, l'incontinence, ou encore la survenue d'une chute n'évoquent pas, à priori, un processus infectieux, d'autant qu'il n'y a pas de fièvre [6]. La sémiologie des infections est très souvent atypique chez le sujet âgé $[1-3,9,12]$. Les facteurs de risque généraux et spécifiques à l'infection étaient dominés par perte d'autonomie $(67,1 \%)$, suivie de l'AEG $(63 \%)$, des portes d'entrées $(53,4 \%)$, de la poly médication $(46,6 \%)$ et des poly pathologies $(23,3 \%)$. Ces facteurs de susceptibilités étaient largement soulignés dans la littérature [10,12]. La majorité de nos patients $(58,9 \%)$ avaient une durée hospitalisation supérieure à sept jours. Ce long délai serait dû aux multiples consultations chez les tradi-praticiens, le milieu hospitalier est le stade ultime du parcours thérapeutique. Dans notre étude, l'âge était un facteur susceptible d'IN. Ces résultats sont conformes à ceux déjà observés par d'autres auteurs qui avaient constaté que le risque d'IN s'accroissait avec l'âge $[1,7]$. Les raisons à l'origine de cette plus grande susceptibilité des personnes âgées sont complexes et probablement multifactorielles [7]. Le processus physiologique de vieillissement se traduit en particulier par des perturbations de la réponse immunitaire humorale et cellulaire $[1,7,10,12]$. Plus généralement, pour chaque organe, ce sont les fonctions de sécrétion, d'épuration et de réparation qui sont perturbées [7]. Par ailleurs, la perte $\mathrm{d}^{\prime}$ autonomie semblait jouer un rôle important dans la survenue d'une IN chez les sujets enquêtés bien que la perte d'autonomie serait responsable d'un syndrome d'immobilisation et peut s'installer avec une diminution des défenses de l'organisme et une prédisposition à l'infection [7,13]. La dénutrition touche 40 à $85 \%$ des sujets âgés $[14,15]$, et multiplie par 4 à 6 le risque infectieux [16]. De la même façon, la présence des portes d'entrées, la poly médication et l'existence d'une poly pathologie menaçant le pronostic vital étaient associés de façon significative à une fréquence accrue d'IN chez ces patients. Les maladies chroniques sont sources d'une diminution des réserves fonctionnelles de l'organisme, qui lors d'une agression aigue peuvent basculer dans un état de décompensation [13]. La poly médication majore le risque d'effets indésirables et favorise la dégradation des capacités de défense chez les personnes âgées $[17,18]$. En dépit de l'insuffisance du plateau technique n'ayant pas permis d'isoler les germes responsables d'IN dans notre contexte, le traitement probabiliste a permis d'obtenir une évolution a été favorable chez la majorité de nos patients $(84,9 \%): 62 / 73$ patients. La létalité était de $15,1 \%$ malgré la prise en charge. Cette létalité était inférieure à celle retrouvée dans une série congolaise (Brazzaville) de 801 cas, les infections représentent la cause immédiate de décès dans $21,1 \%$ des cas [19]. Par contre dans une série 
genevoise de 3000 autopsies gériatriques, les infections représentent la cause immédiate de décès dans $36 \%$ des cas [20]. Cette létalité est liée d'une part à l'espérance de vie de notre population. D'autre part au retard fréquent du diagnostic lié à la symptomatologie atypique ou incomplète et à la rapidité du retentissement de l'infection chez ces personnes fragiles.

Conclusion :

Au total, cette étude a permis avec ses limitations et en particulier la situation transitoire dans le service de médecine interne et maladies infectieuses du centre hospitalier de Kiffa, d'obtenir une description générale sur les facteurs de susceptibilité de risque d'IN chez le sujet âgé. Cependant, Il est difficile de préciser la fréquence réelle des hospitalisations gériatriques dans les centres hospitaliers nationaux. Ce nombre peut paraître faible, il serait plus important si une prospection était faite systématiquement dans les autres services hospitaliers. Cette étude devra être complétée par des audits de pratiques pour mieux identifier les mauvaises pratiques qui sont à l'origine de l'IN. Les résultats de cette étude doivent être complétés par des analyses bactériologiques ciblant l'écologie bactériologique et plus particulièrement les bactéries multi résistants. D'autres études et surtout d'incidence portant sur des infections ciblées (infections du site opératoire, infections urinaires, infections en réanimation, bactéries multi résistantes, etc.) sont nécessaires pour mieux estimer le véritable poids de l'IN dans nos établissements de soins. Enfin, une réflexion sérieuse sur une meilleure planification des hospitalisations de ces patients selon la gravité de la maladie et la disponibilité des soins doit être entreprise pour raccourcir le séjour hospitalier de ces patients le maximum possible.

\section{Conflit d'intérêt}

Pas de conflit d'intérêt

\section{Reference}

1-S. Hammami, M. Chakroun, S. Mahjoub, N. Bouzouaia. Les infections du sujet âgé. Rev Tun Infectiol, $2007 ; 3$ (1) - 8

2-Epidémiologie des infections nosocomiales chez la personne âgée. Le risque infectieux nosocomial chez les personnes âgées. Hygiènes - 1997 - vol. v $-\mathrm{n}^{\circ} 6$

3-Christman D. Infections nosocomiales chez le sujet âgé. Med Hyg. 1990, 48, 3546-3552.

4-Les 100 recommandations pour la surveillance et la prévention des infections nosocomiales. Paris, Conseil Supérieur d'Hygiène Publique de France, 1992.
5-Organisation mondiale de la sante(OMS) : la sante des personnes âgées ;Rapport d'un comité d'expert.OMS-Geneve $1989: 779,112 p$

6-Chevalier B, Margery J, Wade B, Ka S, Diatta B, Gueye M, Mbaye PS, Debonne JM

Perception du risque nosocomial parmi le personnel hospitalier de l'Hôpital Principal de Dakar. Med Trop 2008; 68 : 593-596

7-Simon I, Cocquelin A, Cassou B.L'infection nosocomiale en contexte gériatrique.Presse Med, 2002, 31, 1506-11

8-Ondzotto G, Mban C, Kaba GE, Bissiko F. Morbidite hospitaliere du sujet age dans un service d'oto-rhinolanryngologie. Med. Afr.Noire. $2009 ; 56(10)$.

9-Philippe Letonturier. L'importance des infections chez le sujet âgé enfin reconnue. Presse Méd. 2002 ; 32(31): 1505-20..

10- Bourrée P. Immunity and immunization in elderly. Pathol Biol 2003 ; 51 : 581-85.

11- Merrien D. Particularités des maladies infectieuses chez le sujet âgé. Presse Med 2002; 31 : 1517-20.

12-F. Raschilas, H. Blain, C. Jeandel. Infection et sujet âgé. Traité de Méd. 2006 ; 3-1129.

13-Brynningsen PK, Damsgaard EM, Husted SE. Improved nutitional status in elderly patients 6 months after stroke. L Nutr Health Aging 2007 ; 11 (1) : 75-9.

14- Morley JE. Nutrition in the elderly. Curr Opin Gastroenterol.2002; $18: 240-5$.

15- Zulkowski K. Nutrition and aging : a transdisciplinary approach. Ostomy Wound Manage 2006 ; 52 : 53-7.

16- Raynaud Simon A, Lesourd D. Dénutrition du sujet âgé : conséquences cliniques. Presse Med $2000 ; 29: 2183-90$.

17- Gonthier R, Blanc P, Stierlam F. Should we treat all the disease of the elderly ? Therapy 2004 ; $59: 227-32$.

18- Ferchichi S, Antoine V. Appropriate drug prescribing in the elderly. Rev Med Int. 2004 ; 25 : 582-90

19-J.L. Nkoua, G. Kimbally-Kaky, J. Ekoba, T. Gombet, G. Mouanga-Yidika. Mortalite du sujet âgé, à propos de 801 décès de sujets de 60 ans et plus, Survenus au centre hospitalier et Universitaire de brazzaville. Méd. Afri. Noire ;1992, 39 (1)

20- Michel J P. Susceptibilité des personnes âgées aux infections. Méd Mal infect 1995 ; 25 : 962-3. 
Tableau I : Caractéristiques sociodémographiques, antécédents et facteurs des risques d'IN.

Table I: Sociodemographic characteristics, antecedents and factors likely to IN

\begin{tabular}{|c|c|c|c|}
\hline Paramètres & & Effectif & Pourcentage \\
\hline \multirow{3}{*}{ Tranche d'âge } & 60-70ans & 44 & 60,3 \\
\hline & 71-80ans & 23 & 31.5 \\
\hline & 81-90ans & 6 & 8,2 \\
\hline \multirow[t]{2}{*}{ Sexe } & Masculin & 51 & 69,9 \\
\hline & Féminin & 49 & 30,1 \\
\hline \multirow[t]{2}{*}{ Provenance } & Rural & 50 & 68,4 \\
\hline & Urbaine & 23 & 31,6 \\
\hline \multirow[t]{9}{*}{ Antécédents } & Tuberculose & 15 & 20,5 \\
\hline & Hypertension Artérielle & 10 & 13,6 \\
\hline & Hémiplégie & 7 & 9,5 \\
\hline & Prostatectomie & 6 & 8.2 \\
\hline & Hépatite B chronique & 5 & 6,8 \\
\hline & Asthme & 4 & 5,4 \\
\hline & Diabète & 2 & 2,7 \\
\hline & Insuffisance rénale chronique & 2 & 2,7 \\
\hline & Fibrose pulmonaire & 1 & 1,3 \\
\hline \multirow[t]{5}{*}{ Facteurs de risque } & Perte d'autonomie & 49 & 67,1 \\
\hline & AEG & 46 & 63 \\
\hline & Porte d'entrées & 39 & 53,4 \\
\hline & Poly médication & 34 & 46,6 \\
\hline & Poly pathologies & 17 & 23,3 \\
\hline \multirow[t]{4}{*}{ Sites de l'infection } & Respiratoire & 36 & 49,7 \\
\hline & Uro-génital & 21 & 28,8 \\
\hline & Digestif & 12 & 16,4 \\
\hline & Cutanéomuqueux & 5 & 6,8 \\
\hline
\end{tabular}

Tableau II : Analyse des facteurs susceptibles d'IN

Table II: Analysis of factors likely to IN

\begin{tabular}{|c|c|c|c|c|c|}
\hline \multirow[t]{2}{*}{ Paramètres } & & \multicolumn{2}{|c|}{ Séjour intra hospitalier } & \multirow[t]{2}{*}{ Total } & \multirow[t]{2}{*}{$P$} \\
\hline & & $<7$ jours & $>7$ jours & & \\
\hline \multirow{5}{*}{ Tranches d'âge } & 60-70ans & 12 & 32 & 44 & 0,0062 \\
\hline & 71-80ans & 13 & 10 & 23 & \\
\hline & 81-90ans & 5 & 1 & 6 & \\
\hline & Perte d'autonomie & 27 & 22 & 49 & 0,0004 \\
\hline & AEG & 26 & 20 & 46 & 0,0004 \\
\hline \multirow[t]{3}{*}{ Facteurs de risque } & Porte d'entrées & 24 & 15 & 39 & 0,0001 \\
\hline & Polymédication & 18 & 16 & 34 & 0,04 \\
\hline & Polypathologies & 11 & 6 & 17 & 0,02 \\
\hline
\end{tabular}

